\title{
Bone marrow transplantation for thalassemia: a global perspective
}

\author{
Mohamed Hamed Hussein, ${ }^{1}$ Mohamed El Missiry, ${ }^{1}$ Sadaf Khalid, ${ }^{1}$ Naila Yaqub, ${ }^{2}$ \\ Sarah Khan Gilani, ${ }^{2}$ Itrat Fatima, ${ }^{2}$ Tatheer Zara, ${ }^{2}$ Priya Marwah, ${ }^{3}$ Rajpreet Soni, ${ }^{3}$ \\ Frederic Bernard, ${ }^{1}$ Annunziata Manna, ${ }^{1}$ Cornelio Uderzo, ${ }^{1}$ Lawrence Faulkner ${ }^{1}$ \\ ${ }^{1}$ Cure2Children Foundation, Florence-Italy; ${ }^{2}$ Children's Hospital Pakistan Institute of Medical \\ Sciences, Islamabad-Pakistan; ${ }^{3}$ South East Asia Institute for Thalassemia, Jaipur, India
}

\begin{abstract}
Even though severe thalassemia is a preventable disease, over 100,000 new cases are born yearly, particularly in the Middle East and South-East Asia. Most of these children may not reach adulthood because long-term appropriate supportive care is either inaccessible or unaffordable. Bone marrow transplantation (BMT) remains the only available definitive cure and success rates can be very high in appropriately selected patients, i.e. low-risk younger children with a matched family donor. In these circumstances BMT may be justified medically, ethically as well as financially, in fact, the cost of low-risk BMT is equivalent to that of a few years of non-curative supportive. This manuscript will briefly review the current status of bone marrow transplantation for thalassemia major with particular emphasis on a global prospective and present the experience of the Cure2Children Foundation supporting sustainable and scalable start up BMT programs in low-resource settings. The initial twelve consecutive patients managed in two start up BMT units in Pakistan (Children's Hospital of the Pakistan Institute of Medical Sciences, Islamabad) and India (South East Asia Institute for Thalassemia, Jaipur) were included in this analysis. These initial six patients per each institution where purposely chosen as the focus of this report because they represent the steepest phase of the learning curve. The median age at transplant was 3.9 years, range 0.9 to 6.0 , liver was no greater than $2 \mathrm{~cm}$ from costal margin, and all received matched related BMT. A structured on-site focused training program as well as ongoing intensive on-line cooperation was provided by the Cure2Children team of professionals. At a median follow-up of 7.5 months (range 3.5 to 33.5 months) both thalassemia-free and overall survival are $92 \%$, one patient died of encephalitis-meningitis of unknown cause. No rejections where observed. Neutrophil recovery occurred at a median of 15.5 days
\end{abstract}

Correspondence: Lawrence B. Faulkner

(C) Copyright M.H. Hussein et al., 2013

Licensee PAGEPress, Italy

Thalassemia Reports 2013; 3(s1):e42

doi:10.4081/thal.2013.s1.e42

This article is distributed under the terms of the Creative Commons Attribution Noncommercial License (by-nc 3.0) which permits any noncommercial use, distribution, and reproduction in any medium, provided the original author(s) and source are credited.

Parts of this work were presented at the

"3rd Pan-European Conference on Haemoglobinopathies and Rare Anaemias", Limassol (Cyprus), 24-26 October 2012. (range 13-25) and platelet recovery at 18 days (range 12-27). Toxicities included, fever and neutropenia (10 patients), CMV reactivation (9 patients), acute GVHD grade 3 or less (4 patients), hypertension (4 patients), mild mucositis (3 patients), bacterial sepsis (1 patient). Median number of transfusions was 2 for red cells (range 0 to 7 ) and 5 for platelet transfusions (range 1 to 18). Median post-BMT hospital stay was 49 days (range 33 to 109). No patients developed significant chronic GVHD, one had a suspicion of malaria 8 months post-BMT and one of tuberculosis 11 months post-BMT, both where treated empirically and are doing well. The mean cost of a BMT and follow-up was around $10.164 €(8.952 €$ in Pakistan and $11.377 €$ in India), range 5.618€ to $14.604 €$. In low resource settings matched-related low-risk BMT for thalassemia can be performed with outcomes comparable to richer countries and with a fraction of the costs even from the very beginning of newly developed BMT units and by relatively untrained personnel provided a structured and intensive cooperation program with BMT experts is provided. This observation may have important implications to increase access to cure thalassemia major worldwide and for the startup of new BMT services in low- to middle income countries.

\section{Introduction}

Severe thalassemia accounts for a substantial proportion of childhood mortality, morbidity, health care expenses and donated blood consumption in the Middle East and South-East Asia, were carrier rate ranges of 5 to 30\%.(1) In spite of significant progress in supportive care which, in the best circumstances, may extend life expectancy well into adulthood,(2) most patients in low- to middle-income areas, where thalassemia is most prevalent, do not survive beyond 20 years of age and the risk of blood-borne infections, primarily hepatitis $\mathrm{C}$, remains significant.(3) Even the definition of thalassemia major in low-resource settings is not very clear cut, in fact, the standard criterion of $\geq 8$ red cell transfusions/year may not apply to a setting where pre-transfusion hemoglobin are often as low as 3-4 $\mathrm{g} / \mathrm{dL}$ and where thalassemia intermedia phenotypes frequently occur; in these circumstance the term severe thalassemia, meaning inability to keep a spontaneous hemoglobin greater than $7 \mathrm{~g} / \mathrm{dL}$, i.e. the threshold below which most centers would recommend a regular transfusion regimen2, might better apply.

Bone marrow transplantation (BMT) has been shown to cure thalassemia over 30 years ago(4) and remains the only definitive cure with thalassemia-free survival rates consistently reported over $80 \%$ in selected young low-risk patients with a histocompatible family donor.(5) BMT has also been shown to improve quality of life.(6,7) In areas where thalassemia is endemic, there is a severe shortage of centers for the cure of this diseases; $(8,9)$ this is not only due to lack of financial resources, in fact BMT is less expensive compared to long- 
term supportive care,(10) but other factors may contribute such as lack of specific professional expertise or the perception that transplant unit set up and maintenance costs are prohibitive. In fact, increasing evidence suggests that transplant units performing low-risk BMT's may not require undue financial or professional investments. $(11,12)$

The standard "Pesaro" risk stratification(13) based on chelation history, liver size and presence of fibrosis on liver biopsy, may not apply to chronically undertransfused children in whom hepatomegaly not necessarily reflects severe iron overload and maybe partially corrected with a proper transfusion program pre-BMT. In fact, alternative risk group assignment independent of liver biopsy have been proposed in lowerincome countries based primarily on age and liver size.(14) Children younger than 7 years with liver $\leq 2 \mathrm{~cm}$ below the costal margin may enjoy cure rates greater than $85 \%$.(15)

There is little published data on long-term outcomes, especially in children. Second malignancies after BMT do not seem to be a major issue, possibly because conditioning regimens generally do not incorporate irradiation, and infertility rates are in the $60 \%$ range for both genders.(16)

In spite of large average family size in many thalassemia-prone areas, for the majority of children with thalassemia a matched related donor may not be available. In fact, having a thalassemic child may dissuade parents from taking the risk of having other children with the disease. In the experience of the Cure2Children Foundation on over 400 families screened in Pakistan and India, a fully compatible donorrecipient couple was found in less than $40 \%$ of cases. Unrelated marrow donors have been successfully used, but reported incidence of mortality, rejection and chronic GVHD, however, are slightly higher.(17) Moreover, the extended degree of histocompatibility required makes it very unlikely to find unrelated donors for thalassemia patients most of whom belonging to ethnic groups under-represented in donor registries, not considering the prohibitive costs of donor searches and unrelated marrow procurement. In the European experience, unrelated cord blood has been shown not to be a suitable source of transplantable stem cells for thalassemia major because of high rejection rate and transplant-related morbidity and mortality,(18) moreover, this is a very expensive stem cell product highly dependent of stringent quality control standards.

Partially matched related donors, typically one of the parents, are readily accessible and free. In the only published series, in which extensively T-cell depleted maternal stem cell collections were used in 22 patients, $63 \%$ obtained transfusion independency, transplant-related mortality was $9 \%$, rejection $28 \%$ with no acute or chronic GVHD.(19) The use of postBMT high-dose cyclophosphamide as a means of bidirectional in vivo allodepletion with its associated faster immunological recovery, tolerable delays in hematological engraftment,(20) decreased complexity and costs, could potentially be the most realistically alternative source of transplantable stem cells in lower resource settings but it is still experimental and should be performed only within clinical trials.

\section{International cooperation projects: the Cure2Children Foundation experience}

The Cure2Children Foundation (C2C) was developed by a group of Italian parents who lost their child to cancer. Its primary mission is to increase sustainable access to care for all children with cancer and severe blood disorders. In keeping with its mission, C2C supported the establishment of two new non-profit BMT units: one at the Children's Hospital of the Pakistan Institute of Medical Sciences (PIMS) in Islamabad- Pakistan in 2009 and the other at the Prem Niketan Hospital in Jaipur-India, denominated South-East Asia Institute for Thalassemia (SEAIT) in 2011. The cost of setting up a two-bed BMT unit (at PIMS), only including renovation and basic equipment, was $30,000 €$ and that of a three-bed BMT unit at SEAIT was $100,000 €$

\section{Patient characteristics and methods}

The initial twelve consecutive patients managed in the above two start up BMT units were included in this analysis. These initial six patients per each institution where purposely chosen as the focus of this report because they represent the steepest phase of the learning curve and thus supposedly the ones at highest risk of unfavorable events related to the critical startup phase.

Selection criteria included a diagnosis of severe thalassemia (inability to keep a spontaneous haemoglobin $\geq 7 \mathrm{~g} / \mathrm{dL}$ ), age of 6 years or less, no hepatomegaly (liver $<2 \mathrm{~cm}$ from the right costal margin), availability of a HLA-matched suitable related donor, clear understanding of the risks and benefits of transplant and informed consent for transplantation, no major infectious diseases or other conditions affecting transplant outcome. Hepatitis $\mathrm{C}$ positivity was not considered an exclusion criteria. Eligibility also required serum creatinine, bilirubin and transaminase less than twice normal values, normal chest $\mathrm{x}$-ray and echocardiogram, normal age-appropriate performance scale and institutional commitment to sharing patient data on a daily basis according to privacy regulations. Liver biopsy was not considered mandatory. All procedures in needy patients were offered free of costs.

The two institutions agreed to participate and sign formal agreements with the $\mathrm{C} 2 \mathrm{C}$ specifying the above selection criteria and the overall treatment protocol. Parental consent was obtained in all cases. The first 6 children transplanted in PIMS were between April 2009 and May 2010, and the first 6 children transplanted in SEAIT were between February and June 2012. The median age at transplant was 3.9 years, range 0.9 to 6.0 and all patients received allogeneic BMT from a compatible related donor (11 siblings and one first-degree cousin).

\section{Collaboration methodology}

Management standards for therapy administration, central venous access, severe pancytopenia, immunosuppression, and hospital infection control were addressed by local training, a specific web-based open-source data management software developed by C2C (CDATA) and videoconferencing (Skype). Computerized pre-calculated personalized treatment plans where generated with Microsoft Excel, converted into Adobe PDF files, double-checked and printed locally. This format allowed to minimize transcription errors and have a clear and standard order sheet in compliance with good clinical practices. Nursing personnel was specifically trained on strict hand washing and central line management and was exclusively dedicated to the BMT unit. Nurse/patient ratio was limited to 1:3. On-site focused training was performed by a team of experienced $\mathrm{C} 2 \mathrm{C}$ professionals for the initial 2 transplant procedures and for at least 3 weeks followed by ongoing 24/7 on-line intensive assistance and tutoring via established management/conferencing tools.

\section{Bone marrow transplant procedure}

The following conditioning was utilized Busulfan $3.5 \mathrm{mg} / \mathrm{kg}$ day in 4 divided doses on days -10 to -7 (total dose $14 \mathrm{mg} / \mathrm{kg}$ ), Thiotepa 10 $\mathrm{mg} / \mathrm{kg} /$ day in two divided doses on day -6 (total dose $10 \mathrm{mg} / \mathrm{kg}$ ), and cyclophosphamide $50 \mathrm{mg} / \mathrm{kg}$ day once daily on days -5 to -2 (total dose 
$200 \mathrm{mg} / \mathrm{kg}$ ) followed by the infusion of freshly harvested HLA-compatible marrow on day 0 .

Graft versus host diseases (GVHD) prophylaxis consisted of cyclosporin A starting at $5 \mathrm{mg} / \mathrm{kg}$ i.v. from day -2 to +5 then $3 \mathrm{mg} / \mathrm{kg}$ i.v. from day +6 to +22 to be followed by $10 \mathrm{mg} / \mathrm{kg} /$ day in two daily oral doses for up to day +90 after which it was tapered by $-5 \% /$ week and discontinued at 8-12 months post-BMT unless otherwise indicated. A short methotrexate course consisting of $10 \mathrm{mg} / \mathrm{msq}$ i.v. on days +1 (24 hours after marrow infusion), +3 and +6 , with folinic acid rescue at 24 hours after each methotrexate with 3 doses of $10 \mathrm{mg} / \mathrm{msq}$ i.v. at 8 hour intervals, i.e. at hours $+24 \mathrm{~h}+32 \mathrm{~h}$ and $+40 \mathrm{~h}$. Methylprednisolone at 0.5 $\mathrm{mg} / \mathrm{kg} /$ day i.v. from day -1 to +30 and tapered by $-1 / 3$ every 5 days over 15 days and stopped on day +45 is also used. Patient-specific pre-calculated treatment plans were made available on request. All patients received irradiated blood products.

\section{Infection control, treatment and prophylaxis}

All patients were admitted in single rooms with split air conditioning, private bathrooms and daily cleaning. None of the centers in Pakistan and India had positive pressure gradients or centralized highefficiency particulate air (HEPA) filtration systems. Hand washing was strictly enforced, gloves and gowns were not mandatory.

For anti-helminthic prophylaxis mebendazole $100 \mathrm{mg}$ twice daily for three days was administered before conditioning. For Candida infection prevention fluconazole $3-6 \mathrm{mg} / \mathrm{kg} /$ day as a single dose was used and for herpes virus acyclovir $250-500 \mathrm{mg} / \mathrm{m} 2 /$ dose three times a day from day +1 to +90 . Cytomegalovirus was monitored by real-time PCR. No routine antibacterial prophylaxis was used. For antifungal therapy voriconazole was the first-line drug, for cytomegalovirus activation both ganciclovir and foscarnet were available. Co-trimoxazole at 5 $\mathrm{mg} / \mathrm{kg} /$ dose twice daily for three consecutive days a week was administered for Pneumocystis prophylaxis was administered from the day neutrophil counts reach $500 / \mathrm{mcl}$ to day +90 .

\section{Family support program}

A patient coordinator as well as housing and monthly allowance was provided by $\mathrm{C} 2 \mathrm{C}$ to patients in Pakistan and India as needed. This family support program was implemented throughout the first eight months post BMT.

\section{Statistical analysis}

Kaplan-Meier survival analysis was performed using GraphPad Prism software version 5 March 2007.

\section{Results}

At a median follow-up of 7.5 months (range 3.5 to 33.5 months) both thalassemia-free and overall survival are $92 \%$ (see Figure 1), one patient died of encephalitis-meningitis of unknow cause. No rejections where observed.

Neutrophil recovery occurred at a median of 15.5 days (range 13-25) and platelet recovery at 18 days (range 12-27).

Toxicities included, fever and neutropenia (10 patients), CMV reac- tivation ( 9 patients), acute GVHD grade 3 or less (4 patients), hypertension (4 patients), mild mucositis (3 patients), bacterial sepsis (1 patient). Median number of transfusions was 2 for red cells (range 0 to 7 ), median day of last red cell transfusion was day +14 (range -14 to +152). Median platelet transfusions where 5 (range 1 to 18). Median post-BMT hospital stay was 49 days (range 33 to 109). No patients developed significant chronic GVHD, one had a suspicion of malaria 8 months post-BMT and one of tuberculosis 11 months post-BMT, both where treated empirically and are doing well.

Five patients were $\mathrm{ABO}$ incompatible with their donors (4 patients with minor $\mathrm{ABO}$ incompatibility and one with bidirectional major $\mathrm{ABO}$ incompatibility).

The mean cost of a BMT and follow-up was around $10.164 €(8.952 €$ in Pakistan and 11.377€in India), range 5.618€to $14.604 €($ see Table 1).

\section{Discussion}

To our knowledge this is the first study evaluating BMT units start up outcomes and costs of matched-related transplantation in homogeneous patient groups in low-resource countries.

In keeping with the missions of Cure2Children, the ultimate goal of this project was to increase worldwide access to cure for thalassemia major by exploring the ability to export and reproduce results obtained in affluent countries in low-risk patients with severe thalassemia having a compatible family donor. The regimen employed, i.e. a combination of thiotepa, oral busulfan and cyclophospahamide is well known to be quite tolerable and assure high engraftment rates in younger tha-

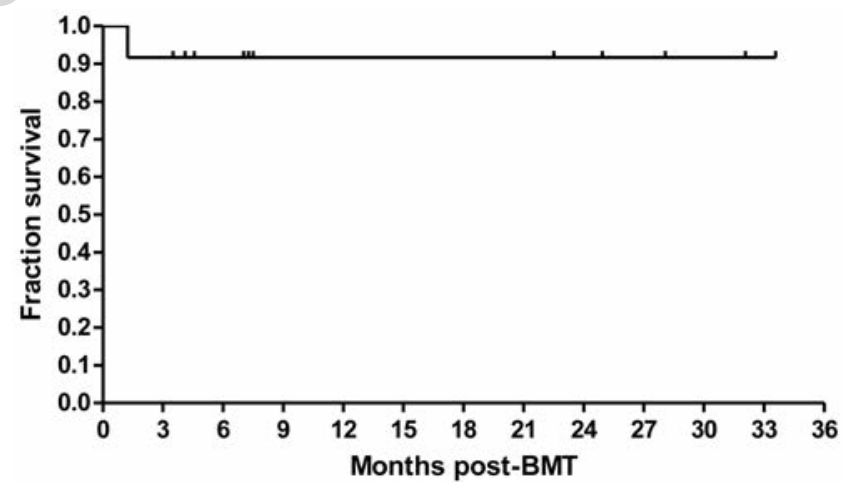

Figure 1. Thalassemia-free survival

Table 1. Mean breakdown costs.

\begin{tabular}{ll}
\hline Pre-transplant evaluation & $€ 357$ \\
Diagnostics & $€ 1,648$ \\
\hline Blood products & $€ 148$ \\
Hospital charges & $€ 1,436$ \\
\hline Surgical (central line and marrow harvest) & $€ 812$ \\
Pharmacy & $€ 2,662$ \\
Professional costs & $€ 2,398$ \\
Follow up costs & $€ 705$ \\
Total BMT costs & $€ 10,164$ \\
\hline
\end{tabular}


lassemic children,(21) i.e. a group known to be at increased risk of rejection.(3)

This objective might be important to increase access to sustainable and scalable tertiary health care by increasing capacity and selfreliance of local centers in underserved regions. In fact, the two centers, PIMS-Pakistan and SEAIT-India, the initial activity if which is summarized in this project, were newly developed services heavily relying on initial on-site whole-team training followed by daily interaction via video-conferencing and web-based data management.

There is general perception that bone marrow transplant centers need complex engineering standards requiring undue investments, even more in poor countries were there might be a greater risk for opportunistic infections. In fact, there is no evidence that the latter is true.(22) Available international guidelines call for placement of allogeneic recipients in highly protected environments with positive pressure gradients, intensive air exchange and filtration,(23) these recommendations however, are not based on clinical trials but rather on limited data largely derived from single-center retrospective studies or expert opinions. In fact, there is increasing evidence that allogeneic transplant patients may be safely cared for in regular hospital rooms 11 or even as outpatients $(24,25)$ and many widely held practices in managing the transplant environment are being reconsidered.

The issue of cost-containment is of paramount importance in settings with very limited resources, where financial restrains directly influence access to cure and thus probability of survival. Any significant expense should be backed up by hard evidence of positive impact on outcomes. Based on this consideration, C2C did not consider mandatory the need for inpatient rooms with pressure differentials and HEPA filtration as well as masks, gowns, or shoe covers. Patient, and his/her caretaker, were admitted to single rooms with private bathrooms and split air conditioning. Strict hand washing of all personnel and visitors was enforced and BMT units were cleaned daily. Infectious complications observed did not seem to be substantially different from those encountered in other countries. In fact, cytomegalovirus reactivation was similarly the main opportunistic infection observed.

BMT programs have a learning curve and become more efficient over time.(16) Bennett et al. showed a learning curve in the BMT at the University of Nebraska where patients receiving BMT for Hodgkin's disease had a $20 \%$ chance of dying after 30 cases and decreased to a $5 \%$ chance after 178 cases; patients with non-Hodgkin's disease had a 33\% chance of dying after 14 cases which also decreased to a $5 \%$ chance after 149 cases26. Furthermore, Bennett et al. determined that changes in costs of BMT decreased significantly over time and the average costs for treatment could be attributed to the decrease in hospitalization days, where length of stay averaged 45 days and decreased to 38 days in four years.(26)

This is the first study to present using telemedicine techniques for close follow-up for BMT patients with a specific web-based open-source data management software developed by C2C (CDATA) and videoconferencing (Skype) (also add patient-specific treatment plans).

Our results show that structured and close collaboration may not only substantially reduce BMT costs by optimizing use of resources but also by minimizing the learning curve effect thus establishing a proof of principle with potential important effects of increasing sustainable and scalable access to cure of severe thalassemia in lower-income regions. Moreover, this experience may be relevant for empowerment of tertiary medical centers in developing countries as well as BMT cost containment globally.

Finally, more accessible BMT may be a way to involve needy families in screening and prevention programs which remains the only realistic way to effectively control thalassemia. Offering prospect of a definitive cure and hope may also be a strong incentive for many families to comply with better supportive care for their affected children.

\section{Conclusions}

Thalassemia is one of the most prevalent chronic life-threatening non-communicable diseases of children in lower-income regions where there is a growing demand for bone marrow transplantation as the only definitive curative modality. In the Cure2Children-supported thalassemia BMT project in developing countries, results in low risk patients have been comparable to those obtained in rich countries with a fraction of the cost, i.e. in the range of $10,000 €$ Moreover, BMT for thalassemia was performed by relatively unskilled personnel within a structured training and collaboration program.

\section{References}

1. Weatherall DJ. The inherited diseases of hemoglobin are an emerging global health burden. Blood. 2010;115(22):4331-4336.

2. Rachmilewitz EA, Giardina PJ. How I treat thalassemia. Blood. 2011;118(13):3479 -3488.

3. Akhtar S, Moatter T. Hepatitis C virus infection in polytransfused thalassemic children in Pakistan. Indian Pediatr. 2004;41(10): 1072-1073.

4. Thomas ED, Buckner CD, Sanders JE, et al. Marrow transplantation for thalassaemia. Lancet. 1982;2(8292):227-229.

5. Angelucci E. Hematopoietic Stem Cell Transplantation in Thalassemia. Hematology. 2010;(1):456-462.

6. Caocci G, Efficace F, Ciotti F, et al. Prospective assessment of health related quality of life in pediatric beta-thalassemia patients following hematopoietic stem cell transplantation. Biol. Blood Marrow Transplant. 2010. Available at: http://www.ncbi.nlm.nih.gov/ pubmed/20870029. Consultato Maggio 2, 2011.

7. Cheuk DK, Mok AS, Lee AC, et al. Quality of life in patients with transfusion-dependent thalassemia after hematopoietic SCT. Bone Marrow Transplant. 2008;42(5):319-327.

8. Aljurf MD, Zaidi SZ, El SH, et al. Special issues related to hematopoietic SCT in the Eastern Mediterranean region and the first regional activity report. Bone Marrow Transplant. 2009;43(1):1-12.

9. Chandy M. Stem cell transplantation in India. Bone Marrow Transplant. 2008;42 Suppl 1:S81-S84.

10. Riewpaiboon A, Nuchprayoon I, Torcharus K, et al. Economic burden of beta-thalassemia/Hb $\mathrm{E}$ and beta-thalassemia major in Thai children. BMC.Res.Notes. 2010;3:29.:29.

11. Kumar R, Naithani R, Mishra P, et al. Allogeneic hematopoietic SCT performed in non-HEPA filter rooms: initial experience from a single center in India. Bone Marrow Transplant. 2009;43(2):115-119.

12. Faulkner L, Yaqub N, Khalid SK, et al. Transplantation in low resource countries. Thalassemia Reports. 2012;1(2s). Available at: http:/www.pagepressjournals.org/index.php/thal/article/view/299.

13. Lucarelli G, Galimberti M, Polchi P, et al. Bone marrow transplantation in patients with thalassemia. NEJM. 1990;322:417-421.

14. Mathews V, George B, Deotare U, et al. A new stratification strategy that identifies a subset of class III patients with an adverse prognosis among children with beta thalassemia major undergoing a matched related allogeneic stem cell transplantation. Biol.Blood Marrow Transplant. 2007;13(8):889-894.

15. Sabloff M, Chandy M, Wang Z, et al. HLA-matched sibling bone marrow transplantation for \{beta\}-thalassemia major. Blood. 2011;117(5):1745-1750.

16. Caocci $\mathrm{G}$, et al. Long-term life experience after allogeneic haematopoietic stem cell transplantation for thalassaemia: a first original report. Bone Marrow Transplantation. 2012;EBMT proceedings:P1034. 
17. La Nasa G, Giardini C, Argiolu F, et al. Unrelated donor bone marrow transplantation for thalassemia: the effect of extended haplotypes. Blood. 2002;99(12):4350-4356.

18. Ruggeri A, Eapen M, Scaravadou A, et al. Umbilical cord blood transplantation for children with thalassemia and sickle cell disease. Biol. Blood Marrow Transplant. 2011;17(9):1375-1382.

19. Sodani P, Isgro A, Gaziev J, et al. Purified T-depleted, CD34+ peripheral blood and bone marrow cell transplantation from haploidentical mother to child with thalassemia. Blood. 2010;115:1296 - 1302.

20. Brunstein CG, Fuchs EJ, Carter SL, et al. Alternative donor transplantation after reduced intensity conditioning: results of parallel phase 2 trials using partially HLA-mismatched related bone marrow or unrelated double umbilical cord blood grafts. Blood. 2011;118(2):282-288.

21. Marziali M, Isgrò A, Gaziev J, Lucarelli G. Hematopoietic Stem Cell Transplantation In Thalassemia And Sickle Cell Anemia. Unicenter Experience In A Multi-Racial Population. Mediterranean Journal of Hematology and Infectious Diseases. 2009. Available at: http:/www.mjhid.org/article/view/5238/html_20. Consultato Aprile $28,2012$.
22. George B, Mathews V, Srivastava A, Chandy M. Infections among allogeneic bone marrow transplant recipients in India. Bone Marrow Transplant. 2004;33:311 - 315.

23. Anon. Guidelines for preventing infectious complications among hematopoietic cell transplant recipients: a global perspective. Bone Marrow Transplant. 2009;44(8):453-558.

24. Svahn B-M, Remberger M, Myrbäck K-E, et al. Home care during the pancytopenic phase after allogeneic hematopoietic stem cell transplantation is advantageous compared with hospital care. Blood. 2002;100(13):4317-4324.

25. Solomon SR, Matthews RH, Barreras AM, et al. Outpatient myeloablative allo-SCT: a comprehensive approach yields decreased hospital utilization and low TRM. Bone Marrow Transplant. 2010;45(3):468-475.

26. Bennett CL, Armitage JL, Armitage G0, et al. Costs of care and outcomes for high-dose therapy and autologous transplantation for lymphoid malignancies: results from the University of Nebraska 1987 through 1991. J. Clin. Oncol. 1995;13(4):969-973.

\section{Oral presentation}

Oral presentation is available online 